\title{
Sterilization by ethylene oxide
}

\author{
J. C. KELSEY ${ }^{1}$ \\ From the Department of Bacteriology, Guy's Hospital Medical School, London
}

Although sterilization by steam under pressure and by hot air are procedures which are now well understood and efficient when properly conducted, there is an increasing number of objects in medical or surgical use which are too heat labile to be so treated. It is for such items as plastic catheters, disposable plastic syringes, and prostheses that ethylene oxide has been proposed as a convenient sterilizing agent. This has been used for years for disinfesting spices and for preventing bacterial spoilage in foodstuffs, but it was only after the publications of Phillips and Kaye (1949) that the possibilities of ethylene oxide as a sterilizing agent began to be appreciated.

Ethylene oxide is a colourless liquid with a boiling point of $10.7^{\circ} \mathrm{C}$. At temperatures above this it is a gas with a characteristic ethereal smell. It forms an explosive mixture when more than $3 \%$ of it is present in air and it is moderately toxic; its toxicity may be compared with that of ammonia. It is very soluble in water and also dissolves in rubber and many plastics. Although the gas forms explosive mixtures with air, if it is mixed with carbon dioxide or with freons in proportions of not more than $12 \%$ by weight the mixture is not inflammable.

Chemically ethylene oxide is a powerful alkylating agent and as such is much used in industry. Its bactericidal effect is probably due to this action: it is lethal to bacteria and to viruses. It is of interest that although vegetative organisms are more easily killed than are spores the difference in resistance is not very great compared with that found in sterilization by hot air or steam. The organism commonly used to test sterilization by ethylene oxide is Bacillus subtilis. var. globigii but Staphylococcus aureus can be almost as resistant. The gas penetrates readily and will pass into the lumen of small tubes, and through fabrics, paper, and many, but not all, plastics.

Unlike sterilization by steam or hot air, where it is comparatively easy to lay down a safe time and temperature to guarantee a sterile load, the use of ethylene oxide is more complex because a greater number of variables need to be considered. These were defined by Phillips and Kaye (1949). As would be expected sterilization depends on time and con-

${ }^{1}$ Present address: Disinfection Reference Laboratory, Central Public Health Laboratory, Colindale Avenue, N.W.9. centration of the gas: when the concentration is doubled the exposure time can be halved. The temperature of the reaction is important and the exposure time is halved for every rise of $17^{\circ} \mathrm{C}$. $\left(30^{\circ} \mathrm{F}\right.$.). It was also shown that the relative humidity can be critical, the optimum being between $20 \%$ and $40 \%$. It would seem, however, that the local humidity of the bacteria to be killed is more important than that of the chamber in which they are exposed. Bacteria which have been desiccated are very difficult to kill. Not only must the ambient humidity be correct but a long time must be allowed for rehydration of the organisms. Recently Opfell, Hohmann, and Latham (1959) have shown that spores dried on to hard surfaces such as glass or plastic were much harder to kill than similar spores dried on to paper. When the spores were suspended in glycerine solution before drying they were easy to kill even on hard surfaces, suggesting that it is the humidity of the immediate environment that is important. These observations have been confirmed by others.

It can be seen from what has been already said that ethylene oxide is a powerful sterilizing agent, but that the number of variables to be considered makes its efficient use rather difficult. In practice a number of techniques have been developed and are in use in various circumstances. The simplest technique, applicable in the laboratory, is to use a desiccator or anaerobic jar as a chamber. The objects to be sterilized are placed in the jar, which is evacuated by a water pump. Ethylene oxide is admitted either as the pure gas or diluted with carbon dioxide or freon, until a manometer indicates that the required percentage has been added. After an exposure period at room temperature or in an incubator the jar is again evacuated to remove the gas, and air is admitted to restore atmospheric pressure before the jar is opened. The humidity of the chamber can be influenced, if not controlled, by placing in it a small dish of water. An alternative is to evacuate the jar and inject into it a measured volume of liquid ethylene oxide using a chilled syringe. This in practice is rather unsatisfactory because the liquid boils at $10^{\circ} \mathrm{C}$. and makes accurate measurement very difficult. These methods using laboratory apparatus can be useful, but they cannot safely be used outside 
a laboratory or where trained staff are not available. For large scale sterilization two rather different processes have been devised on the continent of Europe and in the United States.

In Europe ethylene oxide has been used for many years to disinfest foodstuffs, disinfect hides, and, more recently, to sterilize medical supplies. A large metal chamber is used. After the load has been inserted the door is closed and a vacuum is drawn by an electric pump. A measured volume of liquid ethylene oxide is withdrawn from a cylinder and heated in a special vessel to vaporize it. The resulting gas is introduced into the chamber and raises the pressure towards, but not up to, that of the atmosphere. In some machines the pump re-circulates the air-gas mixture to ensure thorough penetration. After the exposure period the pump is used to vent the gas to atmosphere and to purge the system with one or two changes of filtered air. Because the chamber is always at subatmospheric pressure any leaks will be inward and the risk of explosion or intoxication will be minimized. The process is operated at room temperature and the exposure time is relatively long - usually overnight. In the United States the risks of using pure ethylene oxide are considered to be unacceptable and non-inflammable mixtures are used. In order to compensate for the lower concentration of gas the process is run at a raised pressure, after preliminary evacuation. The exposure time is further shortened by raising the temperature to $60^{\circ} \mathrm{C}$. $\left(140^{\circ} \mathrm{F}\right.$.). Humidity is controlled by injecting known amounts of water or steam.

Both these types of installation are complex and expensive. Schley, Hoffman, and Phillips (1960) have shown that plastic bags or oil drums can be used to improvise a sterilizing chamber, and Freeman and Barwell (1960) have converted an old steam sterilizer. Bracken, Wilton-Davies, and Weale (1960) have described the use of a plastic bag for sterilizing a heart-lung machine. However, because the process needs careful adjustment of several variables there is a tendency to fit sterilizing apparatus with automatic control gear so that reproducible sterilizing cycles are obtained.

Experience has shown that some of the early claims made for ethylene oxide as the perfect sterilizing agent were not justified. Attempts have been made to define a safe process in terms of concentration, time, temperature, and humidity, on the analogy of accepted standards for steam sterilization, but none can be accepted with complete confidence, and none will be given here. Thus Walter and Kundsin (1959) drew attention to the deficiencies of a small gas sterilizer and showed that when operated in accordance with the maker's instructions it did not sterilize test objects.
It is probable that each process must be tested thoroughly with suitable spore preparations in all parts of the load before the process can be regarded as acceptable, and that such tests must be repeated at frequent intervals. Even here a difficulty arises in deciding what is a suitable test object. It is generally agreed that spores of ' $B$. globigii' should be used, but how many should be used, and how should they be prepared? As mentioned earlier, quite different results will be obtained if the spores are dried on paper as compared with plastic, and if $10^{6}$ spores are used instead of $10^{3}$. All that can usefully be said is that the presentation of the spores should resemble the load to be sterilized: plastic for disposable syringes, cloth for dressings, and so on. The numbers of spores used should be related to the probable level of contamination, though this is hard to assess: $10^{6}$ spores per test object are commonly used, though it is probable that numbers as low as $10^{3}$ are adequate.

Most attention has been given to the use of gaseous ethylene oxide for sterilizing medical and surgical supplies. The liquid substance has been used at low temperatures to sterilize liquids such as culture medium and also biological materials such as arterial grafts (Hufnagel et al., 1953) and amniotic membrane (Freeman and Barwell, 1960). Much less experience is available of this application and it remains essentially one for laboratory use and laboratory control.

It is as yet rather early to say precisely what the place of ethylene oxide is in the sterilizing of materials for hospital use. On the commercial scale it is already being used for disposable items such as syringes and catheters, and where adequate bacteriological control is available this seems to be a satisfactory and safe process, although it is possible that the use of ionizing radiation may become econo nically more attractive, as well as more certain in its effects.

There will, however, always be items that need to be sterilized locally at hospital level rather than be bought from commercial sources already sterilized. If the bulk is small the sterilizing can probably be accomplished in the hospital laboratory using im- of provised equipment. If the bulk is greater, either from $N$ larger numbers of small items or single large objects such as heart-lung machines, it is probable that $\omega_{\sigma}$ special sterilizers will be needed with automatic controls and monitoring of temperature, pressure, $\stackrel{\circ}{工}$ and humidity. These machines are expensive to buy $(£ 2,000$ to $£ 3,000$ ) and to operate (about $4 \mathrm{~s}$. per cubic foot of chamber space per run). Because of this and because of the constant need for laboratory $\vec{D}$ control, it is to be hoped that such sterilizers will be $\frac{\rho}{\mathbb{D}}$ centralized in groups or regions where they can be $\varrho$ used both economically and safely. They are not? 
suitable for widespread use in small hospitals or isolated units.

The interested reader is referred to the papers of Phillips and Kaye already mentioned, to the review by Phillips (1957), and to a recent review by Thomas (1960) which contains a very full and up to date bibliography.

\section{REFERENCES}

Bracken, A., Wilton-Davies, C. C., and Weale, F. E. (1960). Guy's Hosp. Rep., 109, 75.
Freeman, M. A. R., and Barwell, C. F. (1960). J. Hyg. (Lond.), 58, 337. Hufnagel, C. A., Rabil, P. J., and Reed, L. (1953). Surg. Forum, 4,162

Opfell, J. B., Hohmann, J. P., and Latham, A. B. (1959). J. Amer. pharm. Ass. (sci. ed.), 48, 617.

Phillips, C. R. (1957). In Reddish, G. F., ed. Antiseptics, Disinfectants, Fungicides and Chemical and Physical Sterilization, 2nd ed., p. 754. Kimpton, London.

- and Kaye, S. (1949). Amer. J. Hyg., 50, 270.

Schley, D. G., Hoffman, R. K., and Phillips, C. R. (1960). Appl. Microbiol., 8, 15.

Thomas, C. G. A. (1960). Guy's Hosp. Rep., 109, 57.

Walter, C. W., and Kundsin, R. B. (1959). J. Amer. med. Ass., 170, 1543. 